\title{
Groups with finitely many non-normal subgroups
}

\author{
By \\ N S Hekster and H W Lenstra, JR
}

1. Introduction. In this paper we describe all groups that have only finitely many non-normal subgroups Examples of such groups are, of course, groups that do not have any non-normal subgroups at all It is well-known that the latter groups can be completely described, as follows

Theorem 1. Let $G$ be a group Then all subgroups of $G$ are normal if and only if $G$ satisfies one of the following two conditions

(1) $G$ is abelian,

(11) there exist groups $A, B$ such that

(a) $G \cong A \times Q \times B$, where $Q$ denotes the quaternion group of order 8 ,

(b) $A$ is an abelian group with the property that every $x \in A$ has finte odd order,

(c) $B$ is an abelian group with $x^{2}=1$ for all $x \in B$

For the proof, see [1, Theorem 1254] A group $G$ is called hamultonian if it satisfies cordition (11) of Theorem 1

For a prime number $p$, denote by $C_{p^{\infty}}$ a multiplicatively written group that is isomorwhic to the group of complex roots of unity of $p$-power order Our main result is as follows

Theorem 2. Let $G$ be a group Then the number of non-normal subgroups of $G$ is finite if and only if $G$ satusfies one of the following three conditions

(1) G is abelian or hamiltontan,

(11) G is finite,

(111) there exist a prime number $p$ and groups $A, B$ such that

(a) $G \cong A \times B$,

(b) A is a finite group of order not divisible by $p$, and it is abelian or hamiltonian,

(c) B has a normal subgroup $C$, contained in the centre of $B$, for which $B / C$ is a finte abelian p-group and $C \cong C_{p^{\infty}}$

For the proof we refer to Section 4 
We can give a formula for the number of non-normal subgroups of the groups occurring 1 Theorem 2 (111) Let $G, p, A, B, C$ be as in. Theorem 2 (111) By [,] we denote the map $B / C \times B / C \rightarrow C$ that is induced by the map $B \times B \rightarrow C$ sending $(g, h)$ to $g^{-1} h^{-1} g h$ If $J_{1}, J_{2}$ are subgroups of $B / C$, then we denote by $\left[J_{1}, J_{2}\right]$ the subgroup of $C$ generated by the image of $J_{1} \times J_{2}$ under [,] Finally, if $D$ is a finite $p$-group, we write $l_{p} D$ for the number of factors $p$ in the order of $D$, so $l_{p} D=(\log \# D) / \log p$

Proposition 3. Let the notation be as just defined, and let $k$ denote the number of subgroups of $A$ Then the number of non-normal subgroups of $G$ equals

$$
k \sum_{J}\left(l_{p}[B / C, J]-l_{p}[J, J]\right) \quad \# J
$$

where $J$ ranges over the set of subgroups of $B / C$

The proof is given in Section 2

It is edsy to see from Proposition 3 that a group $G$ as in Theorem 2 (111) does not have non-normal subgroups at all if and only if $B$ is abelian, this also follows from Theorem 1

The sum appedring in Proposition 3 is clearly divisible by $p$ Assuming that $B$ is not abelian one can, more precisely, show the following If $C$ equals the centre of $B$, then the sum is congruent to $p \bmod p^{2}$, and at least $p(p+1)$, and if $C$ is properly contanned in the centre of $B$, then the sum is congruent to $0 \bmod p^{2}$, and at least $p^{2}(p+2)$ In particular, dny infinite group that has non-normal subgroups at all has at least 6 of them, equality occurs only for the unıque non-abelian group containıng $C_{2^{\infty}}$ as a central subgroup of index 4

Another consequence is the following If the number of non-normal subgroups of a group is a prime number, or the square or the cube of a prime number, then the group is finite

Let $G$ be a group and $\sigma$ an automorphism of $G$ If $H$ is a subgroup of $G$, we say that $\sigma$ fixes $H$ if $\sigma H=H$ The following result is needed in the proof of Theorem 2

Proposition 4. Let $G$ be a group Then the following two assertions are equivalent

(1) $G$ is an infinite abelian group, and it has an automorphism that flxes almost all but not all subgroups of $G$,

(11) there exist a prime number $p$ and groups $A, D$ such that

(a) $G \cong A \times C_{p^{\infty}} \times D$,

(b) A is a finite abelian group of order not divisible by $p$,

(c) $D$ is a non-trivial finte abelian p-group

The same is true if both in (1) and in (11) (b) "abelian" is replaced by "hamiltonian"

The proof is given in Section 3

2. Proof of Proposition 3. Let the notation be as in Proposition 3

Any subgroup of $G$ equals a subgroup of $A$ tumes a subgroup of $B$, so the proof of Proposition 3 immediately reduces to the case that $G=B$, which we now assume We write $\bar{G}=G / C$ 
Let $H$ be a subgroup of $G$, and $\bar{H}$ its image in $\bar{G}$ Clearly we have $[\bar{H}, \bar{H}] \subset H \cap C$, and $H$ is normal in $G$ if and only if $[\bar{G}, \bar{H}] \subset H \cap C$

It follows that the number of non-normal subgroups of $G$ equals $\sum_{J D} n_{J D}$, where the sum ranges over all pairs of subgroups $J \subset \bar{G}, D \subset C$ for which $[J, J] \subset D,[\bar{G}, J] \notin D$, and where $n_{J D}$ is the number of subgroups $H$ of $G$ with $\vec{H}=J$ and $H \cap C=D$

For each $J$, the number of possible $D$ equals $l_{p}[\bar{G}, J]-l_{p}[J, J]$ Now fix $J$ and $D$ Since $D$ is characteristic in $C$ it is normal in $G$ Hence $n_{J} D$ equals the number of subgroups of $G / D$ that map isomorphically to $J$ under the natural map $G / D \rightarrow G / C$ Since $D$ contains $[J, J]$, the inverse image of $J$ in $G / D$ is abelian From $C / D \cong C_{p^{\infty}}$ it follows that this inverse image is isomorphic to $C_{p^{\infty}} \times J$ Thus $n_{J D}$ is the number of subgroups of $C_{p^{\infty}} \times J$ mapping isomorphically to $J$, and this number equals \# Hom $\left(J, C_{p^{\infty}}\right)=\# J$

We conclude that $\sum_{J D} n_{J D}=\sum_{J D} \# J=\sum_{J}\left(l_{p}[\bar{G}, J]-l_{p}[J, J]\right) \# J$, as required This proves Proposition 3

\section{Proof of Proposition 4.}

Lemma 5. Let $G$ be an infinite group, and suppose that $G$ is written as the union of a finite set and a finte collection of subgroups Then the finite set can be omitted from this umon

Proof This is an immediate consequence of a lemma of B H Neumann, which asserts the following If a group is written as the union of finitely many cosets of subgroups, then the cosets occurring in that union belonging to subgroups of infinite index can be omitted For a proof of this lemma, see [2, (4 4), 3, Lemma 417] This proves Lemma 5

Lemma 6. Let $G$ be a group and $\sigma$ an automorphism of $G$ that fixes almost all subgroups of $G$ Then $\sigma$ fixes every infinite subgroup of $G$

P r o of Let $H \subset G$ be an infinite subgroup For every $x \in H-\sigma H$, the subgroup $\langle x\rangle$ generated by $x$ clearly belongs to the finite collection of subgroups $C$ of $G$ with $\sigma C \neq C$ Since for every $C$ there are only finitely many $x \in G$ with $C=\langle x\rangle$, it follows that $H-\sigma H$ is finite Lemma 5 now implies that $H \cap \sigma H=H$, so $H$ is contaned in $\sigma H$ (This can also be seen without Lemma 5) Likewise $H$ is co'ltained in $\sigma^{-1} H$, so $H=\sigma H$, as required This proves Lemma 6

Lemma 7. Let $G$ be an abelian group that has an element of infinite order, and $\sigma$ an automorphism of $G$ that fixes almost all subgroups of $G$ Then $\sigma$ fixes all subgroups of $G$

P r o of Let $T$ be the subgroup of $G$ consisting of all elements of finite order By Lemma 6, one has $\sigma x=x^{ \pm 1}$ for every $x \in G-T$ Since $G / T$ is not the union of two proper subgroups the sign is independent of $x$ But $G-T$ generates $G$, so either $\sigma$ is the identity on $G$ or $\sigma$ maps each $x \in G$ to $x^{-1}$ This implies Lemma 7

Lemma 8. Let $G$ be an abelian group that contains a subgroup of the form $C_{p^{\infty}} \times C_{p^{\infty}}$, where $p$ is a prime number Let $\sigma$ be an automorphism of $G$ that fixes almost all subgroups of $G$ Then $\sigma$ fixes all subgroups of $G$ 
P r o of By Lemma 6, it suffices to prove that $\sigma H=H$ for every fintte subgroup $H$ of $G$ We may clearly assume that $H$ is cyclic Then it is easy to see that there exist subgroups $C_{1}$ and $C_{2}$ of $C_{p^{\infty}} \times C_{p^{\infty}}$, both isomorphic to $C_{p^{\infty}}$, such that $C_{1} \supset H \cap\left(C_{p^{\circ}} \times C_{p^{\infty}}\right)$ and $C_{1} \cap C_{2}=\{1\}$ The infinite subgroups $C_{1} H, C_{2} H$ of $G$ are fixed by $\sigma$, by Lemma 6 , so the same is true for $\left(C_{1} H\right) \cap\left(C_{2} H\right)=H$ This proves Lemma 8

We now prove Proposition 4. We treat the "abelian" and the "hamiltonian" case simultaneously

To prove that (11) implies (1), let $G=A \times C_{p^{\circ}} \times D$ as in Proposition 4(11) It is clear that $G$ is an infinite group that is abelian or hamilonian, as the case may be Let $\phi$ be any non-trivial homomorphism $D \rightarrow C_{p^{\infty}}$ We prove that the automorphism $\sigma$ of $G$ given by $\sigma(a, c, d)=(a, c \phi(d), d)$ fixes almost all but not all subgroups of $G$

If $d \in D$ is such that $\phi(d) \neq 1$, then clearly the subgroup of $G$ generated by $(1,1, d)$ is not fixed by $\sigma$ It remains to prove that $\sigma$ fixes almost all subgroups of $G$ Let $p^{n}$ be the exponent of $D$ Since $C_{p^{\infty}}$ has only finitely many elements of order at most $p^{2 n}$, almost any subgroup $H$ of $G$ has an element $(a, c, d)$ with order $(c)>p^{2 n}$ Taking the $p^{n}$-th power, we see that any such $H$ also contains an element $\left(1, c^{\prime}, 1\right)$ with $c^{\prime} \notin \phi D$. Then $\{1\} \times \phi D \times\{1\} \subset\left\langle\left(1, c^{\prime}, 1\right)\right\rangle \subset H$, and since $\sigma$ acts modulo $\{1\} \times \phi D \times\{1\}$ as the 1dentity this implies that $\sigma$ fixes $H$ This proves that (11) implies (1)

To prove that (1) imphes (11), let $G$ be an infinite abelian or hamilonian group, and let $\sigma$ be an dutomorphism of $G$ that fixes almost all but not all subgroups of $G$

For a prime number $l$, let $G_{l}$ be the subset of $G$ consisting of all elements of finite $l$-power order Since $G$ is abelian or hamiltonian, each $G_{l}$ is a subgroup of $G$, and it is clearly fixed by $\sigma$ Using Lemma 7 we see that $G$ may be identified with the direct sum of all $G_{l}$ For any set $\pi$ of primes, let $G_{\pi}$ be the direct sum of all $G_{l}$ with $l \in \pi$

Let $\pi$ be a set of primes, and $\pi^{\prime}$ its complement, so that $G=G_{\pi} \times G_{\pi}$ Each subgroup of $G$ is the direct sum of a subgroup of $G_{\pi}$ and a subgroup of $G_{\pi}$ It follows that at least one of $G_{\pi}, G_{\pi}$ has a subgroup that is not fixed by $\sigma$, say this is $H \subset G_{\pi}$ Then $H \times G_{\pi}$ is not fixed by $\sigma$, so Lemma 6 implies that $G_{n}$ is finite

This proves that, for any set $\pi$ of primes, one of $G_{\pi}, G_{\pi}$ has a subgroup not fixed by $\sigma$ and the other one is finite

If $G_{l}$ is non-trivid for infinitely many $l$, then we can choose $\pi$ such that both $\pi$ and $\pi^{\prime}$ contain infinitely many such $l$, contradicting what we just proved It follows that almost all $G_{l}$ are trivial Likewise we obtain a contradiction if $G_{l} 1 \mathrm{~s}$ infinite for two distinct primes $l$ Hence there exists a unique prime $p$ such that $G_{p}$ is infinite, and this $G_{p}$ has a subgroup not fixed by $\sigma$ For this prime the group $A=G_{\{p\}}$ is finite, it is either abelian or hamiltonian, and we have $G=A \times G_{p}$

We now first prove that $G_{p}$ is abelian If this is not the case, then we have $p=2$ and $G_{p} \cong Q \times B$, where $B$ is an abelian group of exponent 2 In this group, two elements generate the same subgroup if and only if they are conjugate. Hence the hypothesis that $\sigma$ fixes almost all subgroups implies that $G_{p}$ is the union of a finite set $F$ and $\bigcup_{\phi}\left\{x \in G_{p} \quad \sigma x=\phi x\right\}$, with $\phi$ ranging over the inner automorphisms of $G_{p}$. Since there are only finitely many $\phi$ 's, Lemma 5 implies that the finite set $F$ can be omitted from the union Hence $\sigma\langle x\rangle=\langle x\rangle$ for every $x \in G_{p}$, so $\sigma H=H$ for every subgroup 
$H$ of $G_{p}$, which is a contradiction. This proves that $G_{p}$ is abelian. In particular, $A$ is abelian if and only if $G$ is abelian, and $A$ is hamiltonian if and only if $G$ is hamiltonian.

For a non-negative integer $m$, denote by $G(m)$ the subgroup of all elements of $G_{p}$ of order dividing $p^{m}$. We prove that each $G(m)$ is finite. Suppose that this is not the case. Then we can choose $m$ so large that $G(m)$ is infinite and contains an element $x$ with $\sigma\langle x\rangle \neq\langle x\rangle$. The hypothesis that $\sigma$ fixes almost all subgroups of $G$ implies that $G(m)$ is the union of a finite set $F$ and $\bigcup_{a}\left\{x \in G(m): \sigma x=x^{\alpha}\right\}$, with $a$ ranging over the integers mod $p^{m}$ that are not divisible by $p$. Lemma 5 now implies that the finite set $F$ can be omitted from the union. Hence $\sigma\langle x\rangle=\langle x\rangle$ for every $x \in G(m)$, contradicting the choice of $m$. This proves that all $G(m)$ are finite.

For each $m$, let $C(m)$ be the subgroup $\bigcap_{n \geq 0} G(m+n)^{p^{n}}$ of $G(m)$. Since $G(m)$ is finite, we have $C(m)=G(m+n)^{p^{n}}$ for all $n$ exceeding a bound depending on $m$. This implies that $C(m+1)^{p}=C(m)$ for all $m$, which readily yields that the set $C=\bigcup_{m} C(m)$ is a subgroup of $G_{p}$ that is isomorphic to the direct sum of $t$ copies of $C_{p^{\infty}}^{m}$, for some non-negative integer $t$. Because $C_{p^{\infty}}$ is divisible we have $G_{p} \cong C \times D$ for some subgroup $D$ of $G_{p}$. If $n$ is such that $C(1)=G(1+n)^{p^{n}}$, then $D^{p^{n}}=\{1\}$, so $D \subset G(n)$ and therefore $D$ is finite. But $G_{p}$ is infinite, so we must have $t>0$. By Lemma 8 we have $t<2$. Therefore $t=1$, and $C \cong C_{p^{\infty}}$.

Since $G_{p}$ has a subgroup not fixed by $\sigma$, not every subgroup of $G_{p}$ is characteristic. Hence $G_{p} \neq C$, and $D$ is non-trivial.

This proves Proposition 4.

4. Proof of Theorem 2. The if-part of Theorem 2 is clear in the cases (i) and (ii), and in case (iii) it suffices to refer to Proposition 3.

Before we prove the only if-part we derive a series of auxiliary results.

Lemma 9. Let $G$ be a group with only finitely many non-normal subgroups. Then every infinite subgroup of $G$ is normal.

Proof. This follows from Lemma 6, applied to inner automorphisms of $G$.

Lemma 10. Let $G$ be a group that has only finitely many non-normal subgroups, and $p$ a prime number. Suppose that $G$ contains a normal subgroup $C$ isomorphic to $C_{p}$ for which $G / C$ is a finite cyclic group. Then $G$ is abelian.

Proof. For almost all $x$ in a generating coset of $G$ modulo $C$ the subgroup $\langle x\rangle$ is normal in $G$. Choose such an $x$. Then the natural map $C \rightarrow G /\langle x\rangle$ is surjective, so $G /\langle x\rangle$ is abelian. Therefore the commutator subgroup $G^{\prime}$ of $G$ is contained in $\langle x\rangle$, so $G^{\prime}$ is finite. But $G^{\prime}$ is the homomorphic image $C^{x-1}$ of $C$, so it is divisible as well. Hence $G^{\prime}=\{1\}$. This proves Lemma 10.

Lemma 11. Let $G$ be a group that has only finitely many non-normal subgroups, and $p$ a prime number. Suppose that $G$ contains a subgroup $C$ of finite index that is isomorphic to $C_{p^{\infty}}$. Then $C$ is contained in the centre of $G$. 
P r o of By Lemma 9 the subgroup $C$ is normal Now apply Lemma 10 to subgroups generated by $C$ and a single element of $G$ This proves Lemma 11

Lemma 12. Let $G$ be a group that has only finitely many non-normal subgroups, and $p$ a prime number Suppose that $G$ contains a normal subgroup $C$ that is isomorphuc to $C_{p^{\infty}}$ for which $G / C$ is a finite p-group Then $G / C$ is abelian

P r o of By Lemma 9 we can choose a positive integer $m$ such that every non-normal subgroup of $G$ has order less than $p^{m}$ Denote by $C(m)$ the unique subgroup of $C$ of order $p^{m}$ Then every subgroup of $G$ containing $C(m)$ is normal, so $G / C(m)$ is abelian or hamiltonian But the orders of the elements of $G / C(m)$ are exactly dll powers of $p$, so $G / C(m)$ is not hamiltonian Therefore $G / C(m)$ is abelian, and it follows that $G / C$ is abehan as well This proves Lemma 12

Lemma 13. Let $G$ be an infinite group that has only finitely many non-normal subgroups, and that is netther abehan nor hamiltonian Then $G$ has a normal subgroup $F$ of finte index that is abelian or hamilonian, and that contains a subgroup $H$ that is non-normal in $G$

P r o of We construct a sequence of non-normal subgroups $H_{1}, H_{2}$, , of $G$ and a sequence of normal subgroups $F_{1}, F_{2}$, , of finite index in $G$ with $H_{1} \subset F_{\imath}$ in the following way

Let $H_{1}$ be any non-normal subgroup of $G$, and $F_{1}$ its normalizer in $G$ All conjugates of $H_{1}$ are non-normal in $G$, so they are finite in number Hence $F_{1}$ is of finite index in $G$, and by Lemma 9 it is normal

Suppose, inductively, that $H_{1}, F_{\imath}$ have been constructed If $F_{\imath}$ is abelidn or hamiltonian, then the construction stops, and $F=F_{i}, H=H_{l}$ satisfy the conclusion of the lemma If $F_{t}$ is not abelian or hamiltonian, we let $H_{t+1}$ be any non-normal subgroup of $F_{t}$, and $F_{t+1}$ its normalizer in $F_{t}$ Then $F_{\imath 11}$ is normal of finite index in $G$

To prove that the process stops, it suffices to show that $H_{\imath} \neq H_{J}$ for $\jmath>\imath$ But $H_{\imath}$ is normal in $F_{t}$, whereas $H_{j}$ is not even normal in the subgroup $F_{j-1}$ of $F_{\imath}$ This proves Lemmd 13

We now prove the only if -part of Theorem 2

Let $G$ be a group that has only finitely many non-normal subgroups, and that is not as in (1) or (11) of Theorem 2, $1 \mathrm{e}, G$ is infinite, and neither abelian nor hamiltonian Let $F, H$ be chosen as in Lemma 13, and let $\phi$ be an inner automorphism of $G$ with $\phi H \neq H$ Then $F$ is an infinite group that is abelian or hamitonidn, and the restriction of $\phi$ to $F$ is an automorphism of $F$ that fixes almost all but not all subgroups of $F$ Applving Proposition 4 to $F$ we see that $F$ has a subgroup $C$ of finite index that is isomorphic to $C_{p^{\infty}}$ Then $C$ is of finite index in $G$, so by Lemma 9 each subgroup of $G$ contaning $C$ is normal Therefore $G / C$ is abelian or hamiltonian, and we cdn write $G / C=A \times D$, where $D$ is a finite $p$-group and $A$ is a finite group of order not divisible by $p$ that is abelian or hamiltonian Also, $C$ is contained in the centre of $G$, by Lemma 11

Let $E$ be the unique subgroup of $G$ for which $A=E / C$, and let $a$ be the order of $A$ Since $C$ is uniquely divisible by $a$, the cohomology group $H^{2}(A, C)$ vanishes, so $E$ can be identified with $A \times C$ From $A=\left\{x \in E x^{a}=1\right\}$ we see that $A$ is characteristic in $E$, 
and therefore normal in $G$ The subgroup $B$ of $G$ with $D=B / C$ is also normal in $G$, and it follows that $G=A \times B$ Finally, applying Lemma 12 with $B$ in the role of $G$ we see that $B / C$ is abelian

This completes the proof of Theorem 2

\section{References}

[1] M HALL, $\mathbf{J}_{\mathbf{R}}$, The theory of groups New York 1959

[2] B H NeumanN, Groups covered by permutable subsets J London Math Soc 29, 236-248 (1954)

[3] D J S Robrnson, Finteness condilıons and generalized soluble groups, Part 1 Ergeb Math Grenzgeb (2) 62, Berlin-Hcidelberg-New York 1972

Eingegangen am 591988

Anschuiften der Autoren

N S Hekster

IBM The Netherlands

Paalbergweg 9-11

1105 AG Amsterdam

The Netherlands
H W Lenstrd, Jr

Department of Mathematics

University of Calıfornia

Berkeley, Calıforna 94720

USA 